\title{
HIV/AIDS Model Based on Local Activity of CNN
}

\author{
Xisong Dong \& Gang Xiong (Corresponding author) \\ The State Key Laboratory for Intelligent Control and Management of Complex Systems \\ Institute of Automation, Chinese Academy of Science \\ No. 95 Zhongguancun East Road, Beijing 100190, China \\ Tel: 86-10-6255-4288 E-mail: dongcomic04@163.com,gang.xiong@ia.ac.cn
}

Received: January 17, 2011 Accepted: January 26, 2011 doi:10.5539/jmr.v3n2p168

\begin{abstract}
A Cellular Neural Network (CNN) model of two strain (antiretroviral sensitive and resistant) Human Immunodeficiency Virus (HIV) is established, and then analyzed and simulated based on the local activity of CNN with five local state variables and one port. Numerical simulations exhibit that this CNN model may explain some complex phenomena during antiretroviral therapy which make it possible to judge the curative effect of long-time antiretroviral therapy and social influence. All these imply that the local activity of CNN provides a practical tool for the study of the complex dynamics of some coupled nonlinear systems, especially life systems.
\end{abstract}

Keywords: HIV/AIDS model, Cellular Neural Network (CNN), Local activity, Numerical simulation, Antiretroviral

\section{Introduction}

Coupled nonlinear dynamical systems have been widely studied in recent years. However the dynamical properties-the determination, prediction, and control of these systems are difficult to deal with. Nature abounds with complex patterns and structures emerging from homogeneous media, the local activity is the origin of these complexity (Chua, 1997; Chua 2005). The Cellular Neural Network (CNN), firstly introduced by Chua (Chua, 1988), has been widely studied for image processing, robotic, biological versions, higher brain functions and so on (Chua, 1988). It can model and study many coupled nonlinear systems (Chua, 1999). The local activity of CNN proposed by Chua (Chua, 1997) asserts that a wide spectrum of complex behaviors may exist if the cell parameters of the corresponding CNN are chosen in or nearby the edge of chaos (Chua, 1999; Chua, 2005). This theory has been successfully applied to the research on many complex systems with physical, biological, and chemical backgrounds.

Life systems consist of locally coupled homogeneous media. Virtually, dynamics of life systems are suitable to be described via locally connected CNNs. It may be expected that CNN will become a promising candidate for modeling life phenomena. This theory has been successfully applied to life systems, such as the biochemical model (Min, 2000), coupled excitable cell model (Min, 2002), tumor growth and immune model (Min, 2003), HBV infection model (Min, 2006), T cells ( $\mathrm{Ji}, 2008)$, and so on.

HIV, the Human Immunodeficiency Virus, is the etiological agent of Acquired Immune Deficiency Syndrome (AIDS). Deaths due to AIDS are more than two million people per year recently, making it one of the destructive epidemics in history and the leading cause of death in the world (WHO, 2009). A number of theoretical studies have focused on the mathematical modeling of HIV/AIDS (Mukandavire, 2006; Martcheva, 2007; Bhunu, 2009). But these models were analyzed simply because of the complexity usually. In (Bhunu, 2009), a two strain (antiretroviral sensitive and resistant) HIV/AIDS model with treatment which allows AIDS patients with sensitive HIV-strain to undergo amelioration is presented as a system of nonlinear differential equations with five variables.

In this paper, the model in (Bhunu, 2009) is mapped into a CNN form and the equilibrium points are calculated and analyzed based on the local activity of CNN with five local state variables and one port (Dong, 2009). The bifurcation of this CNN has been calculated and analyzed. Numerical simulations show that this CNN model may explain some complex phenomena during HIV/AIDS treatments. The quantitative understanding of HIV/AIDS dynamics will make it possible to judge the curative effect of long-time antiretroviral therapy and social influence.

\section{Analysis and Simulations Reaction-Diffusion CNN of HIV/AIDS Model}

\subsection{The HIV/AIDS Model and its CNN model}

In (Bhunu, 2009), a two strain (antiretroviral sensitive and resistant) HIV/AIDS model with treatment which allows AIDS patients with sensitive HIV-strain to undergo amelioration is presented as a system of non-linear differential equations, which classifies people into five classes: susceptible $(S)$, antiretroviral sensitive HIV infected $\left(I_{1}\right)$, AIDS individuals with antiretroviral sensitive HIV $\left(A_{1}\right)$, antiretroviral resistant HIV infected $\left(I_{2}\right)$ and AIDS individuals with antiretroviral 
resistant HIV $\left(A_{2}\right)$. The formulation of HIV/AIDS model is

$$
\left\{\begin{array}{l}
\frac{\mathrm{d} S}{\mathrm{~d} t}=a-\left(\frac{b_{1}\left(I_{1}+n A_{1}\right)+b_{2}\left(I_{2}+n A_{2}\right)}{S+I_{1}+A_{1}+I_{2}+A_{2}}+u\right) S \\
\frac{\mathrm{d} I_{1}}{\mathrm{~d} t}=\frac{b_{1}\left(I_{1}+n A_{1}\right) S}{S+I_{1}+A_{1}+I_{2}+A_{2}}-\left(q_{1}+u\right) I_{1}+y e A_{1} \\
\frac{\mathrm{d} A_{1}}{\mathrm{~d} t}=q_{1} I_{1}-\left(u+d_{1}+e\right) A_{1} \\
\frac{\mathrm{d} I_{2}}{\mathrm{~d} t}=\frac{b_{2}\left(I_{2}+n A_{2}\right) S}{S+I_{1}+A_{1}+I_{2}+A_{2}}-\left(q_{2}+u\right) I_{2} \\
\frac{\mathrm{d} A_{2}}{\mathrm{~d} t}=q_{2} I_{2}-\left(u+d_{2}\right) A_{2}+(1-y) e A_{1}
\end{array}\right.
$$

where, individuals are reproduced into the susceptible class at constant rate $a$, and $u$ is a natural death rate in each human subgroup. The antiretroviral sensitive HIV infected becomes AIDS individuals with antiretroviral sensitive HIV $\left(I_{1} \rightarrow A_{1}\right)$ at rate $q_{1}$. Individuals in $A_{1}$ have an additional AIDS-induced death rate $d_{1}$, and are given antiretroviral therapy at rate $e$. A proportion $y$ of individuals in $A_{1}$ given antiretroviral therapy respond well and move into $I_{1}$, and the complimentary proportion $1-y$ develops resistance and enters $A_{2}$. The antiretroviral resistant HIV infected becomes AIDS individuals with antiretroviral resistant HIV $\left(I_{2} \rightarrow A_{2}\right)$ at rate $q_{2}$. Individuals in $A_{2}$ have an additional AIDS-induced death rate $d_{2}$. The force of infection $y_{i}$ is given as

$$
y_{i}=\frac{b_{i}\left(I_{i}+n A_{i}\right) S}{S+I_{1}+A_{1}+I_{2}+A_{2}}, i=1,2
$$

$b_{1}, b_{2}$ is the product of the effective contact rate and the transmission probability of antiretroviral sensitive and resistant HIV infection per contact, respectively. $n>1$ is the modification parameter which models the fact that individuals in the AIDS stage are more infectious than the corresponding HIV infected not yet in the AIDS stage.

The HIV/AIDS CNN model has the form:

$$
\left\{\begin{aligned}
\frac{\mathrm{d} S_{i j}}{\mathrm{~d} t} & =a-\left(\frac{b_{1}\left(I_{1 i j}+n A_{1 i j}\right)+b_{2}\left(I_{2 i j}+n A_{2 i j}\right)}{S_{i j}+I_{1 i j}+A_{1 i j}+I_{2 i j}+A_{2 i j}}+u\right) S_{i j}+D_{1} \nabla^{2} S_{i j} \\
\frac{\mathrm{d} I_{1 i j}}{\mathrm{~d} t} & =\frac{b_{1}\left(I_{1 i j}+n A_{1 i j}\right) S_{i j}}{S_{i j}+I_{1 i j}+A_{1 i j}+I_{2 i j}+A_{2 i j}}-\left(q_{1}+u\right) I_{1 i j}+y e A_{1 i j} \\
\frac{\mathrm{d} A_{1 i j}}{\mathrm{~d} t} & =q_{1} I_{1 i j}-\left(u+d_{1}+e\right) A_{1 i j} \\
\frac{\mathrm{d} I_{2 i j}}{\mathrm{~d} t} & =\frac{b_{2}\left(I_{2 i j}+n A_{2 i j}\right) S_{i j}}{S_{i j}+I_{1 i j}+A_{1 i j}+I_{2 i j}+A_{2 i j}}-\left(q_{2}+u\right) I_{2 i j} \\
\frac{\mathrm{d} A_{2 i j}}{\mathrm{~d} t} & =q_{2} I_{2 i j}-\left(u+d_{2}\right) A_{2 i j}+(1-y) e A_{1 i j}
\end{aligned}\right.
$$

where

$$
\nabla^{2} S_{i j}=S_{i+1 j}+S_{i-1 j}+S_{i j+1}+S_{i j-1}-4 S_{i j}
$$

\subsection{Analysis of Equilibrium Points}

Let Eq.(2) be zero (where $D_{1}=0$ ) and solve it, we can get the three equilibrium points:

$$
\begin{aligned}
Q_{1} & =\left(\frac{a}{u}, 0,0,0,0\right) \\
Q_{2} & =\left(\frac{a\left(d_{2}+q_{2}+u\right)}{A}, 0,0, \frac{a B}{\left(q_{2}+u\right) A}, \frac{a q_{2} B}{\left(d_{2}+u\right)\left(q_{2}+u\right) A}\right) \\
Q_{3} & =\left(\frac{Q_{S}}{Q}, \frac{Q_{I_{1}}}{Q}, \frac{Q_{A_{1}}}{Q}, \frac{Q_{I_{2}}}{Q}, \frac{Q_{A_{2}}}{Q}\right) .
\end{aligned}
$$


where

$$
\begin{aligned}
& A=b_{2}\left(d_{2}+u+q_{2} n\right)-d_{2} q_{2} \text {. } \\
& B=b_{2}\left(d_{2}+u+q_{2} n\right)-\left(d_{2}+u\right)\left(q_{2}+u\right) \text {. } \\
& X=\left(d_{1}+e+u\right)\left(q_{1}+u\right)-e q_{1} y \text {. } \\
& Y=b_{1}\left(d_{1}+e+u+q_{1} n\right)-X \text {. } \\
& Z=\left(b_{1}-b_{2}\right) u^{3}+\left(\left(b_{1}-b_{2}\right)\left(d_{1}++d_{2}+e\right)+n\left(b_{1} q_{1}-b_{2} q_{2}\right)+b_{1} q_{2}-b_{2} q_{1}\right) u^{2}+\left(\left(d_{1}+d_{2}+e\right)\left(b_{1} q_{2}-b_{2} q_{1}\right)\right. \\
& \left.+\left(b_{1}-b_{2}\right)\left(d_{1} d_{2}+d_{2} e+q_{1} q_{2} n\right)+b_{2} e\left(q_{1} y-q_{2} n\right)+n\left(b_{1} d_{2} q_{1}-b_{2} d_{1} q_{2}\right)\right) u+q_{1} q_{2} n\left(b_{1} d_{2}-b_{2} d_{1}\right) \\
& +d_{2}\left(d_{1}-e\right)\left(b_{1} q_{2}-b_{2} q_{1}\right)+b_{2} q_{1}\left(d_{2} e y+e q_{2} n y-e q_{2}\right) y \text {. } \\
& Q_{I_{1}}=a\left(d_{1}+e+u\right) Y Z / X \text {. } \\
& Q_{A_{1}}=a q_{1} Y Z / X \text {. } \\
& Q_{I_{2}}=a q_{1} b_{2} e n(1-y) Y \text {. } \\
& Q_{A_{2}}=\operatorname{aeq}_{1}(1-y)\left(\left(d_{1}+e+u\right)\left(b_{1} u+b_{1} q_{2}-b_{2} u-b_{2} q_{1}\right)+q_{1}\left(b_{2} e y+b_{1} n u+b_{1} n q_{2}\right)\right) Y / X \text {. } \\
& Q_{S}=\frac{\left(q_{2}+u\right)\left(Q_{I_{1}}+Q_{I_{2}}+Q_{A_{1}}+Q_{A_{2}}\right)}{\left(b_{2}-q_{2}-u\right) Q_{I_{2}}+b_{2} n Q_{A_{2}}} \text {. } \\
& Q=\left(b_{1}^{2}-b_{1} b_{2}\right) u^{4}+\left(\left(b_{1}-b_{2}\right)\left(b_{1}\left(2 d_{1}+d_{2}+2 e+q_{1} n\right)-d_{1} q_{1}\right)+b_{1}\left(b_{1}\left(q_{1} n+q_{2}\right)-b_{2}\left(q_{1}+q_{2} n\right)\right)\right) u^{3} \\
& +\left(e q_{1}\left(b_{1} b_{2}(1-n)+b_{1} d_{2}-b_{2} d_{2}\right) y+b_{1}^{2}\left(q_{1}^{2} n^{2}+d_{1}^{2}+e^{2}+2\left(d_{2}+q_{2}+n q_{1}\right)\left(d_{1}+e\right)+2 q_{1} n\left(d_{2}+q_{2}\right)\right.\right. \\
& \left.-2 d_{1} e\right)+q_{1}\left(b_{2}-b_{1}\right)\left(e\left(d_{1}+d_{2}\right)-d_{1} q_{2}\right)+d_{1} q_{1} q_{2}\left(b_{2} d_{1} n-b_{1} d_{1}\right)-b_{1} b_{2}\left(e^{2}+q_{1} q_{2} n^{2}-2 d_{1} e+q_{1} n\left(d_{1}\right.\right. \\
& \left.\left.\left.+q_{2}\right)-2\left(d_{1}+e\right)\left(q_{2} n+d_{2} q_{1}\right)-d_{2} q_{1}(1+n)\right)+d_{1}^{2}\left(b-2 d_{1} e\right)+d_{1}^{2}\left(b_{2} q_{1}-b_{1} b_{2}-b_{1} q_{1}\right)\right) u^{2}+\left(\left(b_{1} b_{2} e q_{1}(1\right.\right. \\
& \left.-n)\left(e+q_{1} n\right)+d_{2} e q_{1}\left(b_{1}-b_{2}\right)\left(d_{1}+e\right)+b_{2} e q_{1}\left(d_{1}+d_{2}\right)\left(b_{1}-q_{1}\right)+b_{1} e q_{1}\left(d_{2} q_{2}+d_{1} q_{1} n-b_{2} d_{1} n\right)\right) y \\
& +b_{1}^{2}\left(\left(d_{2}+q_{2}\right)\left(\left(d_{1}+n q_{1}\right)^{2}+e\left(e+2 d_{1}+2 q_{1} n\right)\right)+d_{1}^{2}\left(b_{2}\left(q_{1}-b_{1}\right)\left(d_{2}+q_{1}+q_{2} n\right)+b_{1} q_{1}\left(d_{2}+q_{2}\right)\right)\right. \\
& -b_{1} b_{2} q_{1} q_{2} n^{2}\left(d_{1}+e+q_{1}\right)+e^{2}\left(b_{1} b_{2} n\left(q_{1}-q_{2}\right)-b_{1} b_{2}\left(q_{1}+d_{2}\right)+d_{2} q_{1}\left(b_{2}-b_{1}\right)\right)+q_{1}^{2}\left(b _ { 2 } \left(d_{1} d_{2}-d_{1} e\right.\right. \\
& \left.\left.\left.+d_{2} e-b_{1} n\left(d_{2}-d_{1}+e-e n\right)\right)-b_{1} d_{1} n\left(d_{2}+q_{2}\right)+n\left(b_{2} d-1 q_{1}-b_{1} d_{2} e\right)\right)\right)+b_{1} e q_{2}\left(b_{1}+d_{2}\right)\left(b_{2}+q_{2}\right) \\
& -b_{1}\left(q_{1}+e\right)\left(d_{2}+n q_{2}\right)+2 b_{1} d-2 e q_{1}\left(b_{2}-b_{1}\right)-q_{1} q_{2} d_{1} e\left(d_{1}+d_{2}\right)+b_{1} b_{2} d_{1} q_{1} n\left(e-d_{2}\right)-b_{1} b_{2} e n q_{1}\left(d_{2}\right. \\
& \left.\left.+q_{1}\right)\right) u-b_{1} d_{1}^{2} d_{2} q_{1} q_{2}+\left(d_{2} e q_{1}\left(d_{1}+e\right)\left(b_{1} b_{2}+b_{1} q_{2}-2 b_{2} q_{1}\right)+q_{1}^{2} e n\left(b_{1} d_{2} q_{1}-b_{2} d_{1} q_{2}+b_{1} d_{2} q_{2}\right)\right) y+q_{1}\left(d_{1}\right. \\
& +e)\left(b_{1} d_{2} n\left(2 b_{1} q_{2}-d_{1} q_{1}-e q_{1}\right)+b_{2} d_{1} q_{1}\left(q_{2} n+d_{2}\right)\right)-b_{1} d_{2} q_{1}\left(b_{2}+q_{2}\right)\left(2 d_{1} e+d_{1}^{2}+e^{2}\right)+q_{1}^{2} e^{2} b_{2} d_{2}\left(1+y^{2}\right) \\
& +q_{1}^{2} n^{2} b_{1} q_{2}\left(b_{1} d_{2}-b_{2} d_{1}\right)+b_{1}^{2} d_{2} e q_{2}\left(2 d_{1}+e\right)+b_{2} d_{1} e q_{1}\left(d_{2} q_{1}-b_{1} q_{2} n\right)
\end{aligned}
$$

The equilibrium $Q_{1}, Q_{2}, Q_{3}$ stand for the disease-free equilibrium, retroviral resistant HIV strain only equilibrium and both HIV strain coexist equilibrium, respectively. But we cannot find the antiretroviral sensitive HIV strain only equilibrium in chapter 3.2.1 in (Bhunu, 2009).

Obviously, the numbers of the equilibrium points could be greater than zero, then, we can get:

$$
\begin{aligned}
& A>0 \quad \Rightarrow \quad \frac{b_{2}\left(d_{2}+q_{2} n+u\right)}{d_{2} q_{2}}>1 \\
& B>0 \quad \Rightarrow \quad R_{2}=\frac{b_{2}\left(d_{2}+q_{2} n+u\right)}{\left(d_{2}+u\right)\left(q_{2}+u\right)}>1 \\
& Y>0 \quad \Rightarrow \quad R_{1}=\frac{b_{1}\left(d_{1}+q_{n}+e+u\right)}{\left(d_{1}+e+u\right)\left(q_{+} u\right)-e q_{1} y}>1
\end{aligned}
$$

where $R_{1}, R_{2}$ are equivalent to " $R_{1}, R_{2}$ " in chapter 3.2.1 in (Bhunu, 2009)(See Page 366).

Consequently, the Jacobian matrix at the equilibrium points $Q_{i}, i=1,2,3$ is

$$
J\left(Q_{i}\right)=\left[J_{1}, J_{2}, J_{3}, J_{4}, J_{5}\right],
$$


where

$$
\begin{aligned}
& J_{1}=\left[\begin{array}{cc}
\frac{b_{1}\left(I_{1}+n A_{1}\right)+b_{2}\left(I_{2}+n A_{2}\right)}{-\left(S+I_{1}+A_{1}+I_{2}+A_{2}\right)} & -\frac{\left(b_{1}\left(I_{1}+n A_{1}\right)+b_{2}\left(I_{2}+n A_{2}\right)\right) S}{\left(S+I_{1}+A_{1}+I_{2}+A_{2}\right)^{2}}-u \\
\frac{b_{1}\left(I_{1}+n A_{1}\right)}{S+I_{1}+A_{1}+I_{2}+A_{2}}-\frac{b_{1}\left(I_{1}+n A_{1}\right) S}{\left(S+I_{1}+A_{1}+I_{2}+A_{2}\right)^{2}} & 0 \\
\frac{b_{2}\left(I_{2}+n A_{2}\right)}{S+I_{1}+A_{1}+I_{2}+A_{2}} & -\frac{b_{2}\left(I_{2}+n A_{2}\right) S}{\left(S+I_{1}+A_{1}+I_{2}+A_{2}\right)^{2}} \\
0
\end{array}\right] . \\
& J_{2}=\left[\begin{array}{c}
\frac{-b_{1} S}{S+I_{1}+A_{1}+I_{2}+A_{2}}+\frac{\left(b_{1}\left(I_{1}+n A_{1}\right)+b_{2}\left(I_{2}+n A_{2}\right)\right) S}{\left(S+I_{1}+A_{1}+I_{2}+A_{2}\right)^{2}} \\
\frac{b_{1} S}{S+I_{1}+A_{1}+I_{2}+A_{2}}-\frac{b_{1}\left(I_{1}+n A_{1}\right) S}{\left(S+I_{1}+A_{1}+I_{2}+A_{2}\right)^{2}}-q_{1}-u \\
\frac{q_{1}}{S+I_{2}\left(I_{2}+n A_{2}\right) S} \\
0
\end{array}\right] . \\
& J_{3}=\left[\begin{array}{c}
\frac{-b_{1} n S}{S+I_{1}+A_{1}+I_{2}+A_{2}}+\frac{\left(b_{1}\left(I_{1}+n A_{1}\right)+b_{2}\left(I_{2}+n A_{2}\right)\right) S}{\left(S+I_{1}+A_{1}+I_{2}+A_{2}\right)^{2}} \\
\frac{b_{1} n S}{S+I_{1}+A_{1}+I_{2}+A_{2}}-\frac{b_{1}\left(I_{1}+n A_{1}\right) S}{\left(S+I_{1}+A_{1}+I_{2}+A_{2}\right)^{2}}+y e \\
-d_{1}-e-u \\
\frac{-b_{2}\left(I_{2}+n A_{2}\right) S}{S+I_{1}+A_{1}+I_{2}+A_{2}} \\
(1-y) e
\end{array}\right] \\
& J_{4}=\left[\begin{array}{c}
\frac{-b_{2} S}{S+I_{1}+A_{1}+I_{2}+A_{2}}+\frac{\left(b_{1}\left(I_{1}+n A_{1}\right)+b_{2}\left(I_{2}+n A_{2}\right)\right) S}{\left(S+I_{1}+A_{1}+I_{2}+A_{2}\right)^{2}} \\
\frac{-b_{1}\left(I_{1}+n A_{1}\right) S}{S+I_{1}+A_{1}+I_{2}+A_{2}} \\
0 \\
\frac{b_{2} S}{S+I_{1}+A_{1}+I_{2}+A_{2}}-\frac{b_{2}\left(I_{2}+n A_{2}\right) S}{\left(S+I_{1}+A_{1}+I_{2}+A_{2}\right)^{2}}-q_{2}-u \\
q_{2}
\end{array}\right] . \\
& J_{5}=\left[\begin{array}{c}
\frac{-b_{2} n S}{S+I_{1}+A_{1}+I_{2}+A_{2}}+\frac{\left(b_{1}\left(I_{1}+n A_{1}\right)+b_{2}\left(I_{2}+n A_{2}\right)\right) S}{\left(S+I_{1}+A_{1}+I_{2}+A_{2}\right)^{2}} \\
\frac{-b_{1}\left(I_{1}+n A_{1}\right) S}{S+I_{1}+A_{1}+I_{2}+A_{2}} \\
0 \\
\frac{b_{2} n S}{S+I_{1}+A_{1}+I_{2}+A_{2}}-\frac{b_{2}\left(I_{2}+n A_{2}\right) S}{\left(S+I_{1}+A_{1}+I_{2}+A_{2}\right)^{2}} \\
-d_{2}-u
\end{array}\right] .
\end{aligned}
$$

\subsection{The Bifurcation Diagram of Equilibrium Points}

Take parameters from Table 1 and let $b_{1}, b_{2} \in(0,1)$ are variables, we can calculate the bifurcation of the CNN model Eq.(2) at the equilibrium points $Q_{1}, Q_{2}$ and $Q_{3}$ based on the local activity of CNN with five local state variables and one port (Dong, 2009), see Fig.2.

In Fig.2, the domains are coded as follows: edge of chaos (locally active and stable) domain (shown red), locally active and unstable domain (shown green) and locally passive domain (shown blue).

\subsection{Simulations and Analysis}

Take parameters from Table 1 and let $b_{1}, b_{2}$ be different numbers, we model the dynamic trajectories of Eq.(2) using MATLAB, see Table 2.

From EQ.(3) and Eq.(4) and take parameters list in Table 1, we can get

$$
\begin{array}{lll}
R_{1}>1 & \Rightarrow & b_{1}>0.0941 \\
R_{2}>1 & \Rightarrow & b_{2}>0.0969
\end{array}
$$

From Fig.2, Table 2, and Eq.(3)-Eq.(6), we can conclude:

1. When $b_{1}, b_{2}$ are both less than $0.0941, b_{1}, b_{2}$ is located in the red domain of Fig.2(a)(the Edge of Chaos domain), $\max \left\{R_{1}, R_{2}\right\}<1$, Eq.(2) converges to the equilibrium point $Q_{1}$ (disease-free) neglecting initial values which implies that there is no any HIV infected and AIDS individuals.

2. When $b_{1}$ or $b_{2}$ is greater than 0.969 , Eq.(2) converges to the equilibrium point $Q_{2}$ or $Q_{3}$, which implies that there are HIV infected and AIDS individuals.

(a) when $b_{1}>0.0969$ and $b_{2}$ is more less than $b_{1}, b_{1}, b_{2}$ are located in the blue domain of Fig.2(c) (the locally passive domain), Eq.(2) converges to the equilibrium point $Q_{3}$ (both HIV strain coexist equilibrium) and $I_{2}<A_{2}$ neglecting initial values which implies that antiretroviral sensitive and resistant HIV infected and 
AIDS individual coexist and the number of antiretroviral resistant AIDS individual is larger than the number of HIV infected. This suggests that the treatment intends to help the community by lengthening the lives of AIDS patients and reducing HIV/AIDS mortality and hence reducing the number of orphans in affected communities.

(b) when $b_{1}>0.0969$, and $b_{2}<b_{1}$ but near $b_{1}, b_{1}, b_{2}$ are located in the red domain of Fig.2(c) (the Edge of Chaos domain), Eq.(2) converges to the equilibrium point $Q_{3}$ and $I_{2}>A_{2}$ neglecting initial values which implies that antiretroviral sensitive and resistant HIV infected and AIDS individual coexist and the number of antiretroviral resistant AIDS individual is less than the number of HIV infected. This suggests that the treatment intends to prolong the incubation period only, but does not reduce infectiousness and take more HIV infections, thus may not benefit the community.

(c) When $b_{1}>0.0969$ and $b_{2} \geq b_{1}, b_{1}, b_{2}$ are located in the red or blue but near red domain of Fig.2(b), Eq.(2) converges to the equilibrium point $Q_{2}$ (retroviral resistant HIV strain only equilibrium) and $I_{2}>A_{2}$ neglecting initial values which implies that there are no antiretroviral sensitive HIV infected and AIDS individual, but antiretroviral resistant HIV infected and AIDS individual still exist. This suggests that the treatment is very effective to antiretroviral sensitive HIV infected and AIDS individual, but the antiretroviral sensitive HIV infected and AIDS individual are more difficult to be treated, which may not benefit the community.

In the following discussions, we select some parameters list in Table 2, the simulation results are shown in Fig.3 and Fig.4. Fig. 3 model the convergence to equilibrium point $Q_{1}$, and Fig.4 model the situation when $b_{1}=0.6$ and $b_{2}$ varies from 0.011 to 0.095 .

\section{Conclusion and Future Works}

A CNN model of two strain (antiretroviral sensitive and resistant) HIV is established, and then analyzed and simulated based on the local activity of CNN with five local state variables and one port (Dong, 2009). Numerical simulations exhibit that this CNN model may explain some complex phenomena during antiretroviral therapy which make it possible to judge the curative effect of long-time antiretroviral therapy and social influence.

Practically, the dynamic behaviors of HIV/AIDS infection and therapy are very complex and puzzling. Then, more and accurate experimental data are needed for modeling the dynamics of HIV/AIDS. Further research for HIV/AIDS dynamics is promising. More complex the model is, more approximate to the reality, but more difficult to be analyzed. The local activity of CNN provides a possible method for some of them.

All these imply that the local activity of CNN provides a practical tool for the study of the complex dynamics of some coupled nonlinear systems. Especially, it may be expected that CNN will become a promising candidate for modeling life phenomena.

\section{Acknowledgement}

This work is supported in part by NSFC 70890084, 60921061, 90920305; CAS 2F09N05, 2F09N06, 2F10E08, 2F10E10.

\section{References}

Bhunu, C. P., Garira, W. \& Magombedze, G. (2009). Mathematical Analysis of a Two Strain HIV/AIDS Model with Antiretroviral Treatment,Acta Biotheor, 57, p. 361-380.

Chua, L. O. (1997). CNN: a version of complexity. Int. J. Bifurcat. Chaos.7(10), p. 2219-2425.

Chua, L. O. (1999). Passivity and Complexity. IEEE Trans. Circ. Syst.-I: Fund. Th. Appl. 46(1), p. 71-82.

Chua, L. O. (2005). Local Activity is the origin of complexity. Int. J. Bifurcat. Chaos. 15(11), p. 3435-3456.

Chua, L. O. \& Yang, L. (1988). Cellular neural networks: Theory and applications. IEEE Trans. Circ. Syst. 35(10), p. 1257-1290.

Dong, X. S., Min, L. Q. \& Ji, Y. (2009). Some Analytical Criteria for Local Activity of One-port CNN with Five State Variables. World Sci-Tech R\&D, 31(1), p149-154.

Ji, Y., Min, L. Q. \& Meng, Y. (2008). Analytical Criteria for the Local Activity of Three-Port CNN with Five State Variables:Analysis and Application. The 9th Int. Conf. for Young Computer Scientists. p. 2759-2765.

Martcheva, M., Ianelli, M. \& Xue, Z. L. (2007). Subthreshold coexistence of strains: the impact of vaccination and mutation. Math Biosci. Engin.4(2), p. 287-317.

Min, L. Q. \& Dong, X. S. (2006). A viral dynamic CNN model of hepatitis B viral infection. Dynam. Contin. Discr. Impuls. Syst. Series B: Appl. Algor. 13(c), p. 35-50.

Min, L. Q., Meng, Y. \& Chua, L. O. (2008). Applications of local activity theory of CNN to controlled coupled oregonator systems. Int. J. Bifurcat. Chaos. 18(11), p. 3233-3297. 
Min, L. Q. \& Yu, N. (2000). Analytical criteria for local activity of CNN with two port and applications to biochemical model. J. Univ. Sci. Technol. Beijing,7(4), p. 305-314.

Min, L. Q. \& Yu, N. (2002). Some analytical criteria for local activity of two-port CNN with three or four state variables: Analysis and applications.Int. J. Bifurcat. Chaos. 12(5), p. 931-963.

Min, L. Q., Wang, J. T., Dong, X. S. \& Chen, G. R. (2003). Some analytical criteria for local activity of three-port CNN with four state variables: analysis and applications. Int. J. Bifurcat. Chaos.13(8), p. 2189-2239.

Mukandavire, Z. \& Garira, W. (2006). HIV/AIDS model for assessing the effects of prophylactic sterilizing vaccines, condoms and treatment with amelioration. J. Biolo. Syst. 14(3), p. 323-355.

UNAIDS/WHO. (2009). 09 AIDS epidemic update, 2009.

Table 1. Model parameters and their interpretations

\begin{tabular}{ccc}
\hline Parameter & Symbol & Value(/year) \\
\hline Recruitment rate & $a$ & 0.029 \\
Product of effectively rate and probability & $b_{1}, b_{2}$ & $0.011-0.95$ \\
Modification parameter & $n$ & 1.02 \\
Natural mortality rate & $u$ & 0.02 \\
Natural rate of progression of HIV & $q_{1}, q_{2}$ & 0.1 \\
Proportion of effectively treated & $y$ & 0.2 \\
Treatment rate for AIDS cases & $e$ & 0.33 \\
AIDS related death rate & $d_{1}, d_{2}$ & $0.333-0.4$ \\
\hline
\end{tabular}


Table 2. Cell parameters and corresponding dynamic properties of the CNN of HIV/AIDS model

\begin{tabular}{|c|c|c|c|c|c|c|c|}
\hline No. & $b_{1}$ & $b_{2}$ & Domain in Fig.1 & Equilibrium point & Relation of $I_{2}$ and $A_{2}$ & $R_{1}$ & $R_{2}$ \\
\hline 1 & 0.011 & 0.011 & red in Fig.2(a) & $Q_{1}$ & $I_{2}=A_{2}$ & 0.1140 & 0.1169 \\
\hline 2 & 0.011 & 0.05 & red in Fig.2(a) & $Q_{1}$ & $I_{2}=A_{2}$ & 0.1140 & 0.5315 \\
\hline 3 & 0.011 & 0.094 & red in Fig.2(a) & $Q_{1}$ & $I_{2}=A_{2}$ & 0.1140 & 0.9993 \\
\hline 4 & 0.05 & 0.011 & red in Fig.2(a) & $Q_{1}$ & $I_{2}=A_{2}$ & 0.5181 & 0.1169 \\
\hline 5 & 0.05 & 0.05 & red in Fig.2(a) & $Q_{1}$ & $I_{2}=A_{2}$ & 0.5181 & 0.5315 \\
\hline 6 & 0.05 & 0.094 & red in Fig.2(a) & $Q_{1}$ & $I_{2}=A_{2}$ & 0.5181 & 0.9993 \\
\hline 7 & 0.094 & 0.011 & red in Fig.2(a) & $Q_{1}$ & $I_{2}=A_{2}$ & 0.9740 & 0.1169 \\
\hline 8 & 0.094 & 0.05 & red in Fig.2(a) & $Q_{1}$ & $I_{2}=A_{2}$ & 0.9740 & 0.5315 \\
\hline 9 & 0.094 & 0.094 & red in Fig.2(a) & $Q_{1}$ & $I_{2}=A_{2}$ & 0.9740 & 0.9993 \\
\hline 10 & 0.011 & 0.097 & red in Fig.2(b) & $Q_{2}$ & $I_{2}>A_{2}$ & 0.1140 & 1.0312 \\
\hline 11 & 0.097 & 0.097 & red in Fig.2(b) & $Q_{2}$ & $I_{2}>A_{2}$ & 1.0051 & 1.0312 \\
\hline 12 & 0.097 & 0.011 & blue in Fig.2(c) & $Q_{3}$ & $I_{2}<A_{2}$ & 1.0051 & 0.1169 \\
\hline 13 & 0.2 & 0.011 & blue in Fig.2(c) & $Q_{3}$ & $I_{2}<A_{2}$ & 0.2072 & 0.1169 \\
\hline 14 & 0.2 & 0.08 & blue in Fig.2(c) & $Q_{3}$ & $I_{2}<A_{2}$ & 0.2072 & 0.8505 \\
\hline 15 & 0.2 & 0.094 & blue in Fig.2(c) & $Q_{3}$ & $I_{2}<A_{2}$ & 0.2072 & 0.9993 \\
\hline 16 & 0.2 & 0.095 & red in Fig.2(c) & $Q_{3}$ & $I_{2}>A_{2}$ & 0.2072 & 1.0099 \\
\hline 17 & 0.2 & 0.19 & red in Fig.2(c) & $Q_{3}$ & $I_{2}>A_{2}$ & 0.2072 & 2.0198 \\
\hline 18 & 0.2 & 0.2 & red in Fig.2(b) & $Q_{2}$ & $I_{2}>A_{2}$ & 0.2072 & 2.1261 \\
\hline 19 & 0.2 & 0.5 & blue in Fig.2(b) & $Q_{2}$ & $I_{2}>A_{2}$ & 0.2072 & 5.3153 \\
\hline 20 & 0.2 & 0.95 & blue in Fig.2(b) & $Q_{2}$ & $I_{2}>A_{2}$ & 0.2072 & 10.099 \\
\hline 21 & 0.6 & 0.011 & blue in Fig.2(c) & $Q_{3}$ & $I_{2}<A_{2}$ & 6.2171 & 0.1169 \\
\hline 22 & 0.6 & 0.36 & blue in Fig.2(c) & $Q_{3}$ & $I_{2}<A_{2}$ & 6.2171 & 3.8270 \\
\hline 23 & 0.6 & 0.4 & red in Fig.2(c) & $Q_{3}$ & $I_{2}>A_{2}$ & 6.2171 & 4.2523 \\
\hline 24 & 0.6 & 0.56 & red in Fig.2(c) & $Q_{3}$ & $I_{2}>A_{2}$ & 6.2171 & 5.9532 \\
\hline 25 & 0.6 & 0.6 & blue in Fig.2(b) & $Q_{2}$ & $I_{2}>A_{2}$ & 6.2171 & 6.3784 \\
\hline 26 & 0.6 & 0.95 & blue in Fig.2(b) & $Q_{2}$ & $I_{2}>A_{2}$ & 6.2171 & 10.099 \\
\hline 27 & 0.9 & 0.011 & blue in Fig.2(c) & $Q_{3}$ & $I_{2}<A_{2}$ & 9.3256 & 0.1169 \\
\hline 28 & 0.9 & 0.56 & blue in Fig.2(c) & $Q_{3}$ & $I_{2}<A_{2}$ & 9.3256 & 5.9532 \\
\hline 29 & 0.9 & 0.58 & blue in Fig.2(c) & $Q_{3}$ & $I_{2}>A_{2}$ & 9.3256 & 6.1658 \\
\hline 30 & 0.9 & 0.87 & blue in Fig.2(c) & $Q_{3}$ & $I_{2}>A_{2}$ & 9.3256 & 9.2486 \\
\hline 31 & 0.9 & 0.9 & red in Fig.2(b) & $Q_{2}$ & $I_{2}>A_{2}$ & 9.3256 & 9.5676 \\
\hline 32 & 0.9 & 0.95 & red in Fig.2(b) & $Q_{2}$ & $I_{2}>A_{2}$ & 9.3256 & 10.099 \\
\hline
\end{tabular}

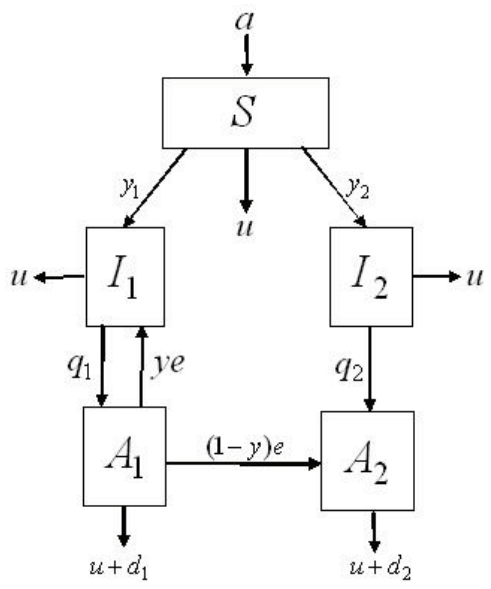

Figure 1. Structure of the HIV/AIDS model 
(a)

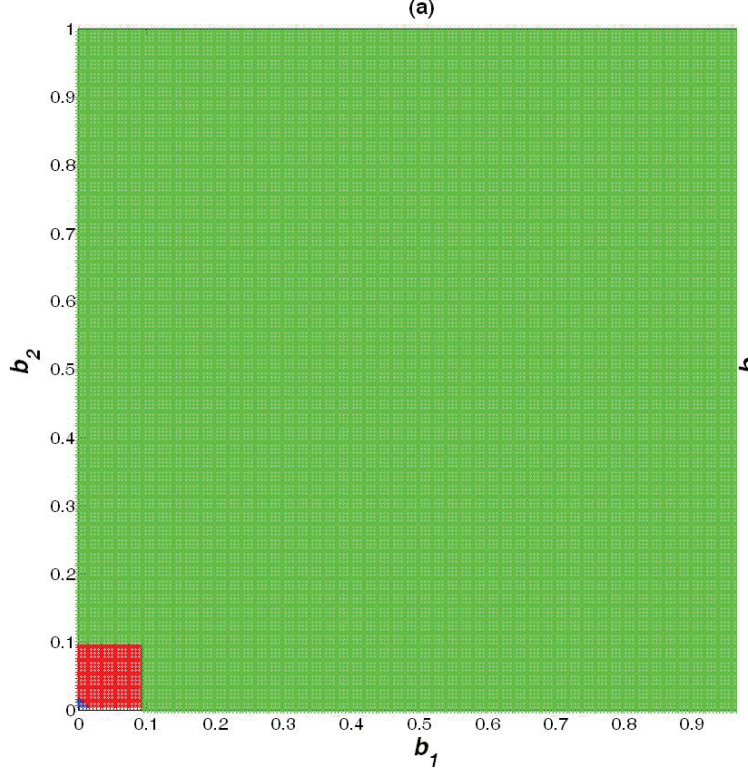

(b)

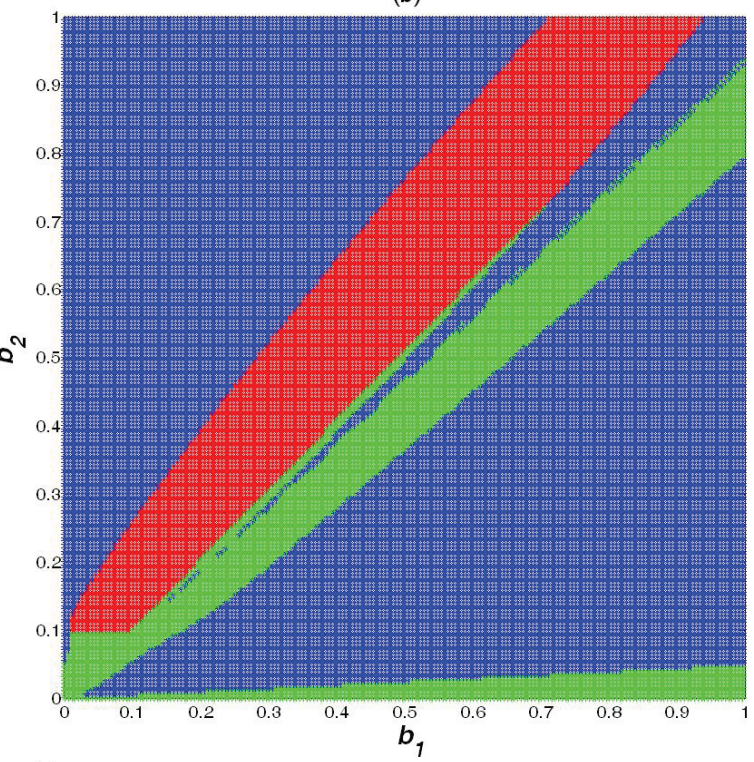

(c)

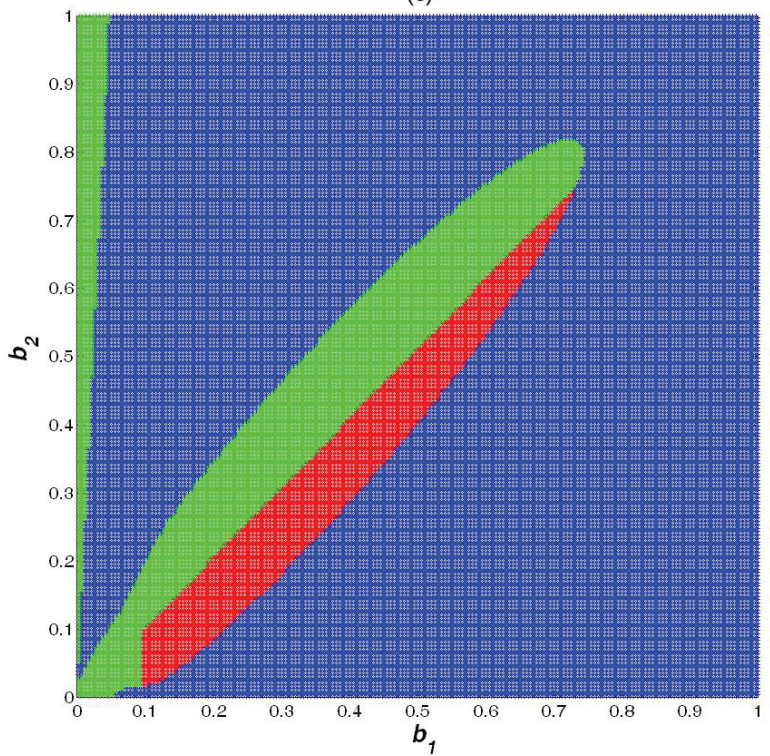

Figure 2. Bifurcation diagrams of Eq.(3), when $b_{1}, b_{2} \in(0,1)$, at the equilibrium point: (a) $Q_{1}$,(b) $Q_{1}$, (c) $Q_{1}$

(a)

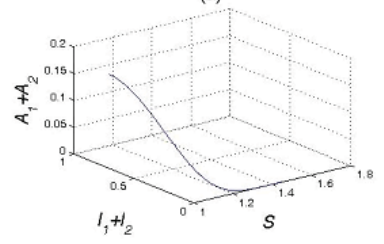

(c)

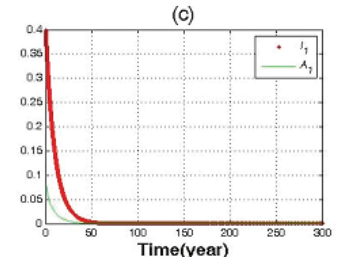

$b_{1}=0.01, \quad b_{2}=0.011$ (b)

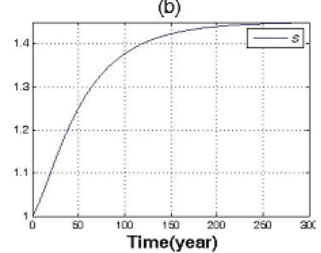

(d)

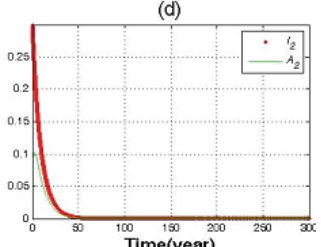

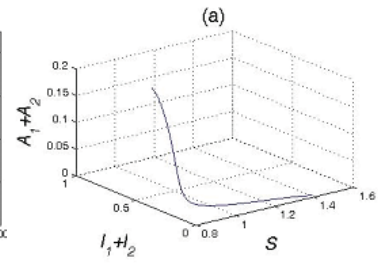

(c)

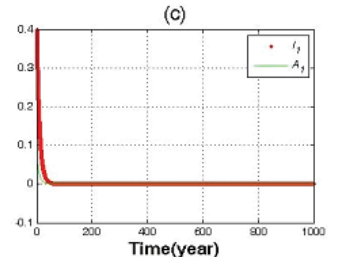

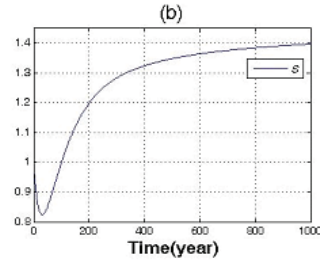

(d)

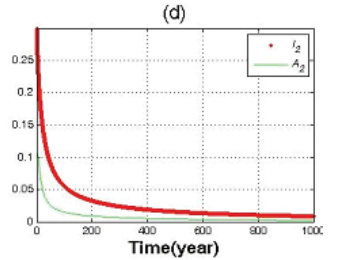



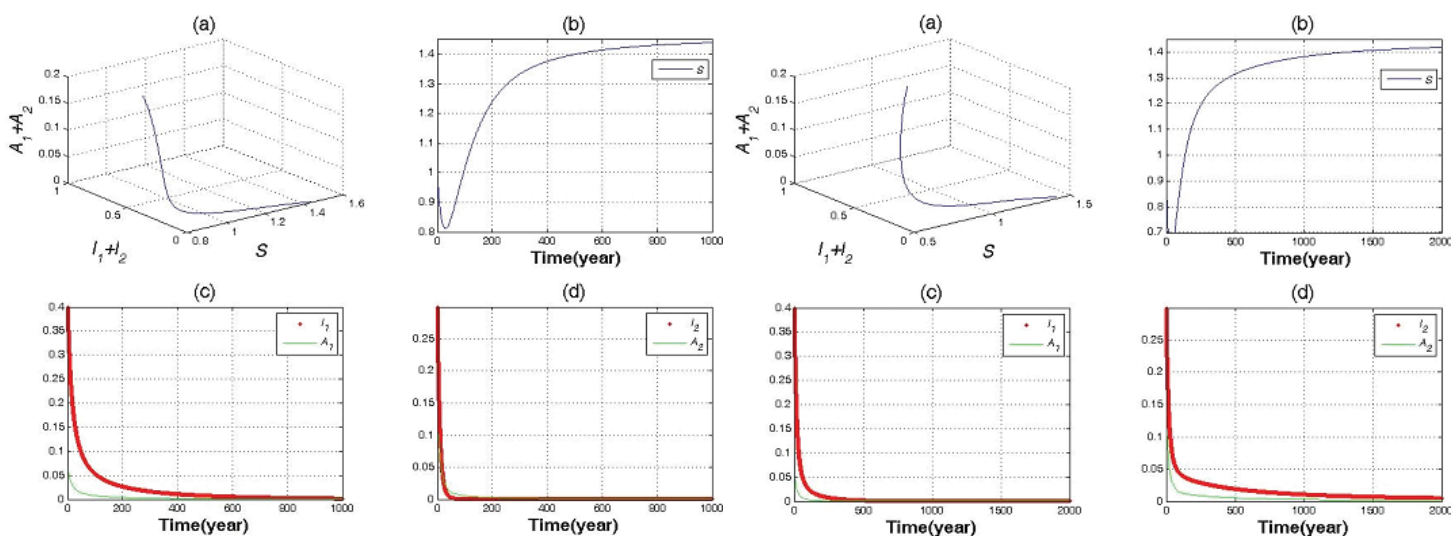

(d)
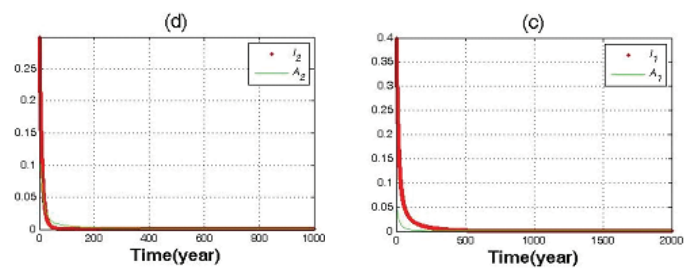

$b_{1}=0.094, \quad b_{2}=0.094$

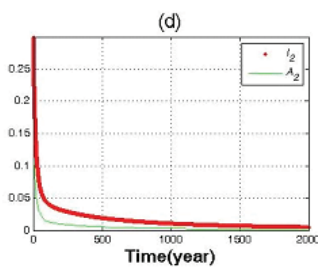

Figure 3. The trajectories of Eq.(2) converging to $Q_{1}$
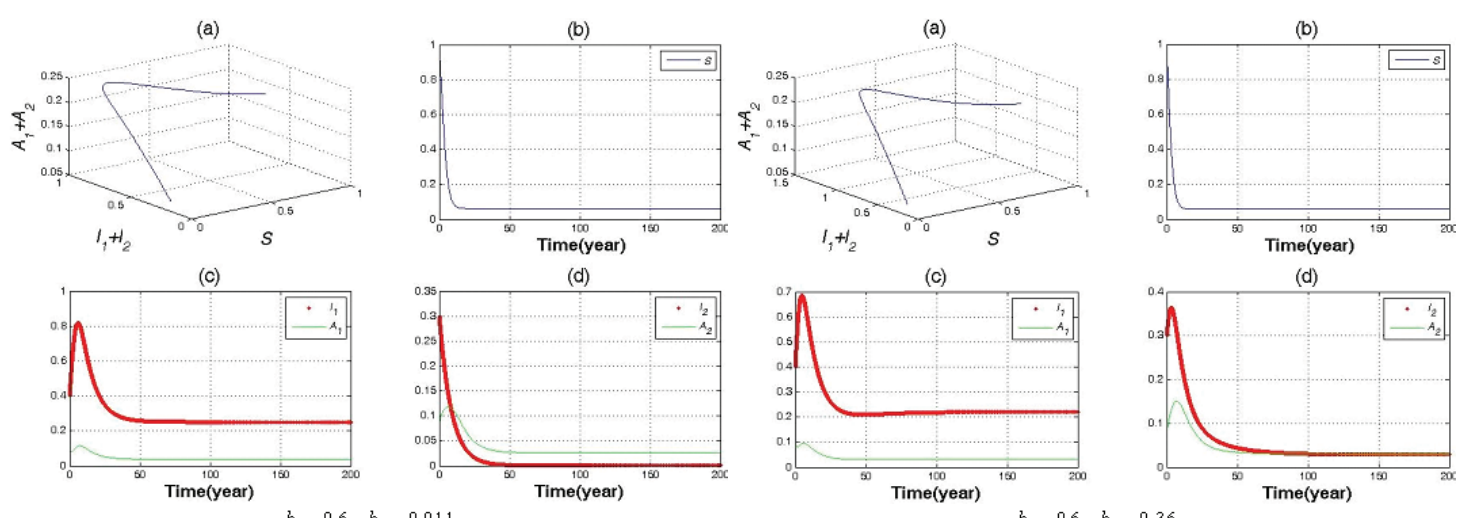

(d)
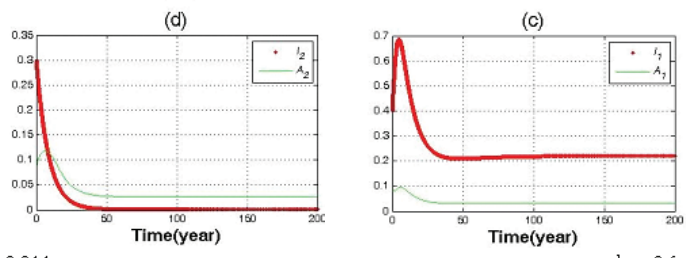

(d)

(a)
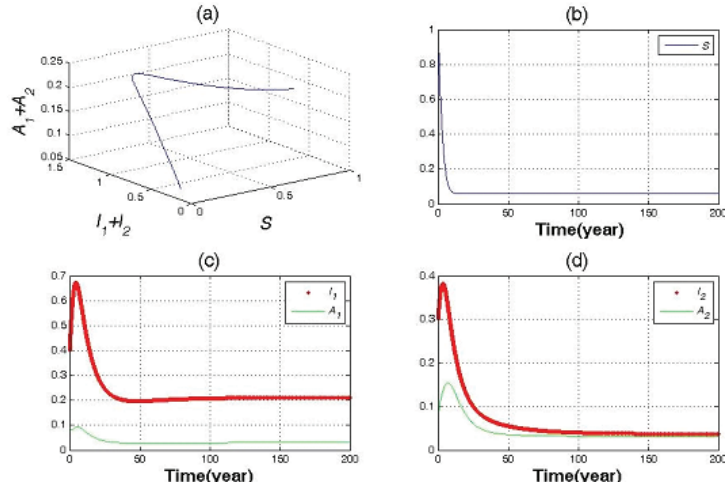

(d)

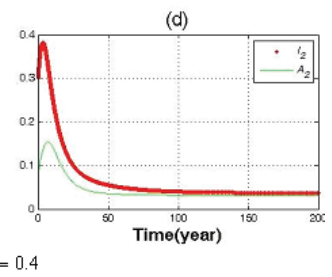

(a)

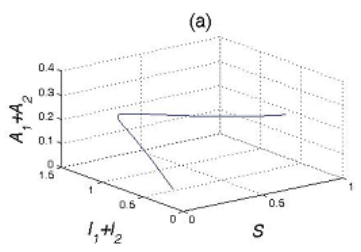

(c)

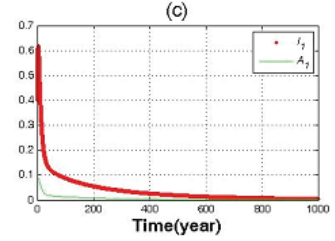

$b_{1}=0.6, \quad b_{2}=0.6$

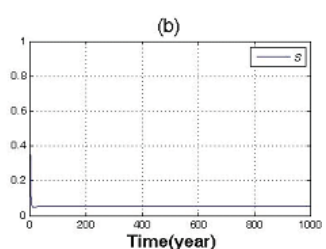

(d)

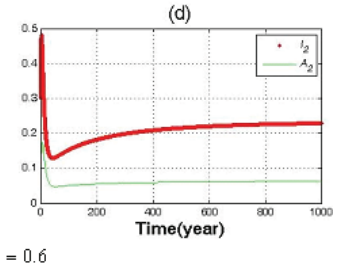

(a)
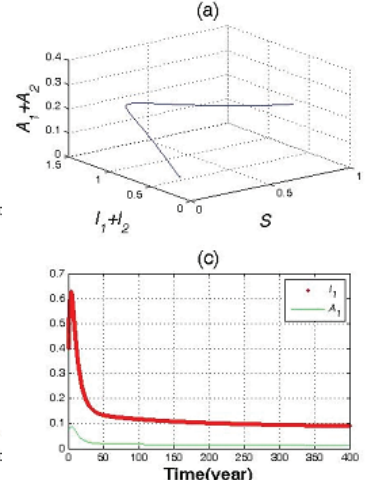

(a)

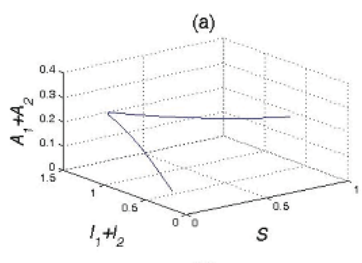

(c)

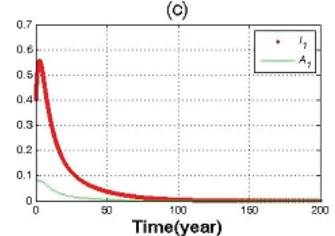

$b_{1}=0.6, \quad b_{2}=0.95$

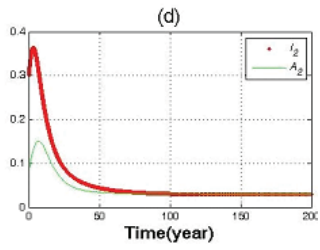

(b)

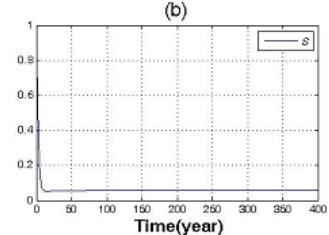

(d)

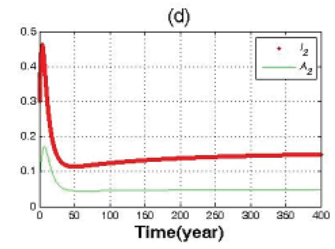

(b)

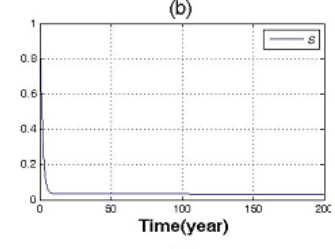

(d)

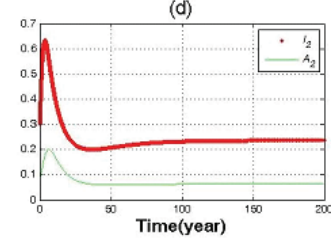

Figure 4. The trajectories of Eq.(2) when $b_{1}=0.6$ 